\title{
Outcome of Combining Ventral Hernia Repair with Abdominoplasty or Mesh-abdominoplasty in Multiparous Women
}

\author{
Mahmoud Sakr ${ }^{1, *}$, Mahmoud Habib ${ }^{2}$, Hossam Hamed ${ }^{2}$, Hassan Kholosy ${ }^{1}$ \\ ${ }^{1}$ Department of Surgery, Faculty of Medicine, Alexandria University, Egypt \\ ${ }^{2}$ Department of Surgery, KOC Ahmadi Hospital, Ahmadi, Kuwait \\ *Corresponding author: mah_sakr@yahoo.com
}

Received November 21, 2018; Revised December 29, 2018; Accepted January 14, 2019

\begin{abstract}
Objectives: to determine the outcome of ventral hernia repair during abdominoplasty and mesh-abdominoplasty regarding cosmesis, recurrence and post-operative complications. Subjects and Methods: The present retrospective study included 78 multiparous women with ventral hernia and abdominal wall deformity. Age ranged between 28 and 59 years with a mean of $41.37 \pm 11.18$ years. Half of the patients suffered from episodes of colicky abdominal pain and 34 (43.6\%) had chronic low back pain. Forty patients (with defect $<3 \mathrm{~cm}$ ) underwent standard abdominoplasty (Group 1) and 38 (with defect $>3 \mathrm{~cm}$ ) underwent mesh-abdominoplasty (Group 2), both after primary suture hernia repair and midline fascial plication. The follow-up period ranged from 22 months to 11 years with a mean of 62.5 months. Results: Both groups were comparable regarding their demographic and clinical parameters. Patients with abdominoplasty had significantly $(\mathrm{p}=0.0193$ ) more para-umbilical hernias (PUHs) than those with mesh-abdominoplasty ( $80 \%$ vs $55.3 \%$, respectively), but had less incisional or recurrent PUHs. All repaired hernias did not recur except for one patient in each group. No mortality or major complications were encountered. Wound complications occurred in 7 patients (17.5\%) in Group 1 vs 10 (26.3\%) in Group 2 ( $\mathrm{p}=0.346)$. Recurrence of abdominal wall deformity and the need for a second refashioning procedure were significantly higher among patients who underwent abdominoplasty alone ( $\mathrm{p}=0.011$ and $\mathrm{p}=0.0139$, respectively). Conclusions: (1) During abdominoplasty, ventral hernia repair and midline plication can be performed in defects $<3 \mathrm{~cm}$ with no increase in hernia recurrence rate, (2) in patients with defects $>3 \mathrm{~cm}$, additional mesh reinforcement is indicated and (3) Prolene mesh-abdominoplasty for multiparous women with severe musculo-aponeurotic laxity and ventral hernia, yields lower recurrence of abdominal deformity and less refashioning procedures with minimal complications than abdominoplasty alone.
\end{abstract}

Keywords: ventra hernia, Prolene mesh, abdominoplasty, mesh-abdominoplasty, multiparous, recurrence, body contour

Cite This Article: Mahmoud Sakr, Mahmoud Habib, Hossam Hamed, and Hassan Kholosy, "Outcome of Combining Ventral Hernia Repair with Abdominoplasty or Mesh-abdominoplasty in Multiparous Women." Global Journal of Surgery, vol. 7, no. 1 (2019): 5-11. doi: 10.12691/js-7-1-2.

\section{Introduction}

Ventral hernias, which include umbilical, para-umbilical, epigastric, and incisional hernias are a relatively common finding among patients undergoing abdominoplasty. These individuals usually seek the abdominal contouring surgery after repeated pregnancies, which are known to cause abdominal wall laxity and deformity [1-5] difficult to reverse with dieting and muscular exercise $[6,7,8]$. A lot of literature has discussed repair of these hernias alone, using open or laparoscopic techniques. However, the literature is lacking as to the discussion of these hernia repairs when combined with abdominoplasty. These hernias may be repaired without utilizing a reinforcing mesh, especially with simultaneous rectus diastasis plication or may require mesh-abdominoplasty after midline plication [9].

The present retrospective study was conducted to determine the outcome of ventral hernia repair during abdominoplasty and mesh-abdominoplasty regarding cosmesis, post-operative complications and recurrence of hernia or abdominal deformity.

\section{Patients and Methods}

\subsection{Study Protocol}

The medical records of all multiparous women (2 children or more) who underwent standard abdominoplasty or 
Prolene mesh-abdominoplasty between January 2006 and January 2017 were retrospectively reviewed. Procedures were performed at two different hospitals (where the first author worked); namely, Alexandria University Main Hospital in Egypt and Kuwait Oil Company Ahmadi Hospital in Kuwait. The same protocol was applied in both hospitals regarding selection criteria, pre-operative work-up, operative technique, post-operative care and follow-up. The protocol of the study was approved by the ethical committee in each hospital.

\subsection{Criteria of Selection and Data Collection}

General contraindications to abdominoplasty were considered in patient selection, including severe co-morbid conditions, morbid obesity (body mass index [BMI] >40), future plans for pregnancy, a history of thrombo-embolic disease, and unrealistic patient expectations. During the 11-year period of the study, 381 multiparous women were admitted for correction of their abdominal wall deformity. According to Bozola and Psillakis classification [10], 246 patients (64.57\%) had a grade IV abdominal wall deformity). They underwent conventional abdominoplasty. Of those, 40 patients had an associated ventral hernia (Group 1). Of the 135 (35.43\%) multiparous women who had a grade $\mathrm{V}$ deformity, 84 underwent mesh-abdominoplasty. Of those, 38 patients had a ventral hernia (Group 2).

Multiparous women who underwent post-bariatric abdominoplasty or torsoplasty (belt lipectomy) $(n=18)$, and those with incomplete medical records $(n=21)$ were not included in the study. All data were collected prospectively and included history-taking, physical examination targeted at addressing abdominal deformity, severity (grade) of laxity of musculo-aponeurotic abdominal wall, presence of associated diastasis of recti and/or ventral hernia, pre-operative work-up, operative technique, post-operative care, complications, recurrence of hernia or abdominal deformity and re-operative procedures.

\subsection{Study Population}

All patients with ventral hernias $(n=78)$ were multiparous women with 2-9 children and Grades IV/V abdominal wall deformity [10]. Age ranged between 28 and 59 years with a mean of $41.37 \pm 11.18$ years. Their BMI ranged between 21.40 and 39.81 with a mean of $32.20 \pm 6.89$. Physical characteristics of the study population are shown in Table 1 . In addition to abdominal wall deformity in all patients, 39 (50.0\%) patients suffered from episodes of colicky abdominal pain, 34 (43.6\%) had chronic low back pain. Forty patients (51.28\%) underwent standard abdominoplasty with no mesh enforcement (Group 1), while 38 patients (48.72\%) underwent mesh-abdominoplasty (Group 2), both after primary suture hernia repair and midline fascial plication. Medical co-morbidities were documented in 29 patients (37.1\%); the most common were type-II diabetes mellitus $(n=9)$, hypertension (HTN) $(n=8)$ and both conditions $(n=5)$. Forty-five patients (57.7\%) underwent previous abdominal surgery; most commonly Cesarean section (range 1-6) $(n=27)$ followed by laparoscopic cholecystectomy $(n=9)$ and para-umbilical hernia (PUH) hernia repair $(n=5)$.

\subsection{Pre-operative Planning and Preparation}

Laboratory investigations included complete blood picture, coagulation profile, liver and renal function tests, lipid profile and blood glucose level. A plain chest X-ray and abdominal ultrasound were done for all patients and an ECG for those above the age of 50 years. As per protocol, all patients received a third-generation cephalosporin intravenously with induction of anesthesia. Prophylactic anti-coagulation therapy was not used and prevention of thrombo-embolism was done by volume loading with lactated Ringer's solution, post-operative leg exercises and early ambulation.

\subsection{Operative Technique}

An informed written consent was obtained from each patient prior to surgery. Pre-operative markings were done with the patient in the erect posture. Patients received general anesthesia with the patient supine. Before commencing the procedure, all patients received suctionassisted lipectomy sparing the subcostal areas.

Steps of the procedure are depicted in Figure 1 (A-F). The lower line of the incision was a sinuous curve or a lazy-W and the upper was a downward curving line just above the umbilicus to meet both ends of the lower incision. The lipo-cutaneous flap was elevated to the xiphoid and medial costal margins. The umbilicus was resected when deemed necessary (as in PUH) to be followed at a later step by neo-umbilicoplasty otherwise (as in epigastric hernia), the umbilicus was incised circumferentially through the skin, and subcutaneous dissection was beveled away from the umbilicus to the fascia preserving the umbilical stalk and peri-umbilical perfusion. The sac was dissected, carefully opened and contents reduced. The defect was then closed with primary repair using non-absorbable sutures.

Fascial plication using a single layer of interrupted figure-of-eight buried Prolene sutures was performed without tension from the xiphoid to the umbilical stalk and downward to the pubis so that the abdominal wall was made tight. This single layer of plication included the advancement of the lateral border of the anterior rectus on both sides and reached the anterior border of the external oblique fascia in some patients according to the degree of musculo-fascial laxity. In Group 2 patients, a well spread Prolene mesh with an average size of $30 \times 30 \mathrm{~cm}$ was then fixed to the external oblique fascia using a fascial stapler or interrupted 2/0 Prolene sutures. The prolene mesh was then trimmed to suit the area of the aponeurotic sheath to be covered.

The dissected abdominal flap was then pulled downwards to establish the final line for skin excision and the site for future umbilicus relocation. A new umbilicus was formed or, in case of preserved umbilicus, a small oval (or inverted V-shape) incision was made in the dissected flap for exteriorization of the umbilical stalk. In either case, the relocation site chosen for the umbilicus was either midway between the two iliac crests [11] or at a ratio of 1.6:1 from the xiphi-sternum to the symphysis pubis [12]. The umbilical stalk was fixed to the fascia using interrupted vertical mattress $3 / 0$ Prolene sutures. Closure was completed with 3/0 Vicryl sutures and 5/0 Monocryle. 

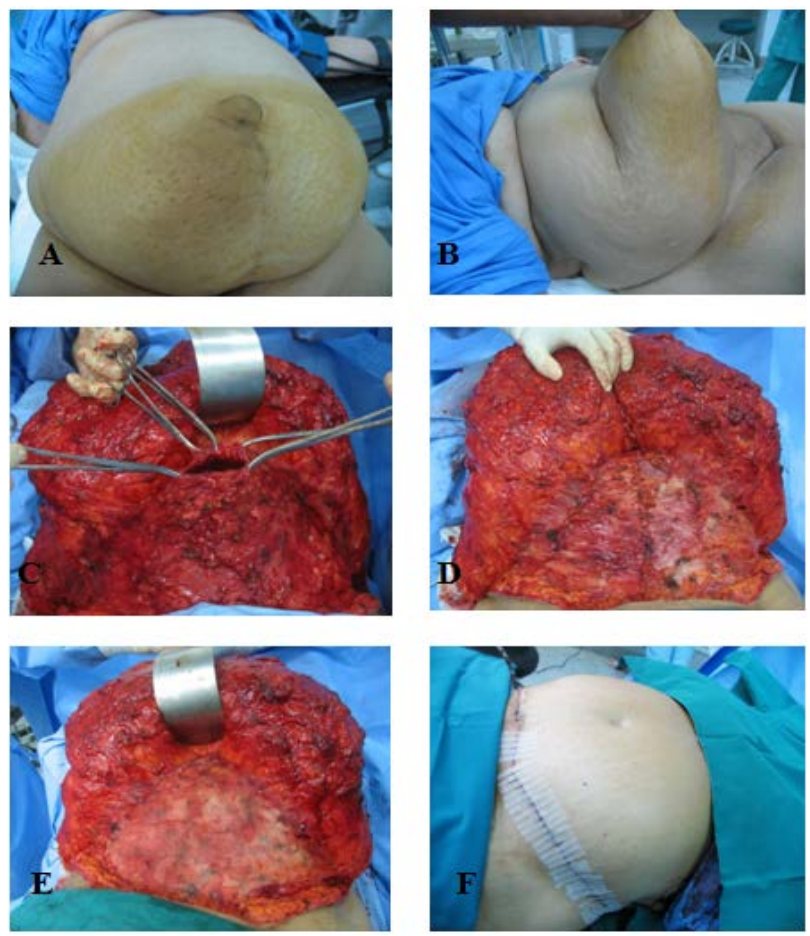

Figure 1. (A-F) Steps of mesh-hernioplasty (Group 1). (A) Preoperative front view of a 39-year-old lady who gave birth to 5 children, (B) Preoperative lateral view with the patient supine, (C) Dissection of the flap till the xiphoid showing the large defect after reduction of the contents and excision of the sac, (D) Midline fascial plication, (E) Prolene mesh application and fixation, (F) Neo-umbilicoplasty, final closure with suction drainage.

Two large-size suction drains were inserted and exteriorized through separate stabs in the skin to one side of the pubis. The wound was then closed using interrupted 2/0 Vicryl sutures and the skin approximated using continuous 3/0 subcuticular Prolene suture starting from the lateral ends and proceeding to the midline. Steristrips were used at the end of the procedure and an abdominal binder was applied.

\subsection{Post-operative Care and Follow-up}

Post-operative respiratory and leg exercises and early ambulation were applied by all patients on the first post-operative day. No antibiotics were routinely administered post-operatively. Drains were removed on the $3^{\text {rd }}-5^{\text {th }}$ post-operative day, after which patients were discharged with clear instructions to wear an abdominal binder and refrain from strenuous physical activity including sports, both for 3 months.

The follow-up period ranged from 22 months to 11 years with an average of 62.5 months, to assess body contour, post-operative complications and recurrence of the hernia or abdominal wall deformity, which was categorized into 5 grades with grade I meaning epigastric bulge only and grade $\mathrm{V}$ total recurrence of musculo-aponeurotic laxity [13]. Seroma was documented when found clinically and required aspiration(s).

\subsection{Statistical Analysis}

The SPSS/PC version 21 computer software (Prentice-Hall; Chicago, IL, USA) was used for statistical analysis. The
Student's t-test was used to compare the mean values of both groups. The Chi square $\left(\chi^{2}\right)$ test with Yates correction was used for comparison between categorical values. A "p" value of $<0.05$ was considered statistically significant.

\section{Results}

The characteristics of the two groups with ventral hernia constituting the study population are summarized in Table 1. As may be seen, both groups were comparable with no statistical differences regarding demographics, BMI, main complaints, medical co-morbidities and history of previous abdominal surgery. Types of ventral hernia encountered in both groups are listed in Table 2. Patients in Group 1 (abdominoplasty) had significantly more PUHs as compared to those in Group 2 (mesh-abdominoplasty) (80\% versus $55.3 \%$, respectively) $\left(\mathrm{X}^{2}=5.475, \mathrm{p}=0.0193\right)$ but had less incisional hernias $(5.0 \%$ versus $18.3 \%$, respectively). Moreover, 5 patients (13.2\%) in Group 2 presented with recurrent $\mathrm{PUH}$ as compared to none in Group 1.

Table 1. Clinical parameters of multiparous women with ventral hernia and severe abdominal wall laxity*

\begin{tabular}{|c|c|c|c|}
\hline Parameters & $\begin{array}{l}\text { Abdomino-plasty } \\
(\mathrm{N}=40)\end{array}$ & $\begin{array}{c}\text { Mesh-Abdomino- } \\
\text { plasty }(\mathrm{N}=38)\end{array}$ & $\begin{array}{c}\text { Total } \\
(\mathrm{N}=78)\end{array}$ \\
\hline $\begin{array}{l}\text { No. of } \\
\text { children }\end{array}$ & $2-8$ & $3-9$ & $2-9$ \\
\hline \multicolumn{4}{|l|}{ Age (years) } \\
\hline Range & $28-53$ & $29-59$ & $28-59$ \\
\hline Mean \pm SD & $40.96 \pm 11.73$ & $41.77 \pm 10.62$ & $41.37 \pm 11.18$ \\
\hline \multicolumn{4}{|l|}{ BMI $\left(\mathrm{Kg} / \mathrm{m}^{2}\right)$} \\
\hline Range & $21.4-39.7$ & $25.1-39.8$ & $21.4-39.8$ \\
\hline Mean \pm SD & $30.77 \pm 7.13$ & $33.67 \pm 6.21$ & $32.2 \pm 6.89$ \\
\hline \multicolumn{4}{|l|}{ Complaints } \\
\hline $\begin{array}{l}\text { Abdominal } \\
\text { deformity }\end{array}$ & 40 (100\%) & 38 (100\%) & $78(100 \%)$ \\
\hline Colicky pain & 12 (30.0\%) & $27(71.1 \%)$ & $39(50.0 \%)$ \\
\hline $\begin{array}{l}\text { Chronic low } \\
\text { back pain }\end{array}$ & $11(27.5 \%)$ & 23 (60.5\%) & $34(43.6 \%)$ \\
\hline \multicolumn{4}{|l|}{$\begin{array}{l}\text { Medical co- } \\
\text { morbidity }\end{array}$} \\
\hline Absent & $26(65.0 \%)$ & $23(60.5 \%)$ & $49(62.9 \%)$ \\
\hline Present & $14(35.0 \%)$ & $15(39.5 \%)$ & $29(37.1 \%)$ \\
\hline$D M$ & $4(10.0 \%)$ & $5(13.2 \%)$ & $9(11.5 \%)$ \\
\hline$H T N$ & $3(7.5 \%)$ & $5(13.2 \%)$ & $8(10.2 \%)$ \\
\hline$D M+H T N$ & $3(7.5 \%)$ & $2(5.2 \%)$ & $5(6.4 \%)$ \\
\hline $\begin{array}{l}\text { Bronchial } \\
\text { asthma }\end{array}$ & $2(5.0 \%)$ & 0 & $2(2.6 \%)$ \\
\hline Peptic ulcer & $1(2.5 \%)$ & $2(5.2 \%)$ & $3(3.8 \%)$ \\
\hline $\begin{array}{l}\text { Hypo- } \\
\text { thyroidism }\end{array}$ & $1(2.5 \%)$ & $1(2.7 \%)$ & $2(2.6 \%)$ \\
\hline \multicolumn{4}{|l|}{$\begin{array}{l}\text { Pervious abd. } \\
\text { surgery }\end{array}$} \\
\hline Present & $25(62.5 \%)$ & $20(52.6 \%)$ & $45(57.7 \%)$ \\
\hline Absent & 15 (27.5\%) & $18(47.4 \%)$ & $33(42.3 \%)$ \\
\hline
\end{tabular}

BMI: body mass index, PUH: para-umbilical hernia, DM: diabetes mellitus, HTN: hypertension, abd: abdominal

*All differences between the 2 groups were not statistically significant $(\mathrm{p}>0.05)$. 
Table 2. Types of ventral hernia in multiparous women in both groups (Group 1, abdominoplasty and Group 2 meshabdominoplasty) $(\mathrm{N}=78)$

\begin{tabular}{|l|c|c|c|}
\hline $\begin{array}{l}\text { Type of Ventral } \\
\text { Hernia }\end{array}$ & $\begin{array}{c}\text { Abdomino- } \\
\text { plasty } \\
\text { (N=40) }\end{array}$ & $\begin{array}{c}\text { Mesh- } \\
\text { abdomino- } \\
\text { plasty (N=38) }\end{array}$ & $\begin{array}{c}\text { Test of } \\
\text { significance }\end{array}$ \\
\hline PUH* & $32(80.0 \%)$ & $21(55.3 \%)$ & \\
Incisional hernia & $2(5.0 \%)$ & $7(18.3 \%)$ & \\
Recurrent PUH & 0 & $5(13.2 \%)$ & $\mathrm{X}^{2}=5.475 *$ \\
Epigastric hernia & $3(7.5 \%)$ & $3(7.9 \%)$ & $\mathrm{p}=0.0193$ \\
Diastasis of recti & $2(5.0 \%)$ & $2(5.3 \%)$ & \\
$\begin{array}{l}\text { Laparoscopic port } \\
\text { hernia }\end{array}$ & $1(2.5 \%)$ & 0 & \\
\hline
\end{tabular}

* Statistically significant $(\mathrm{p}<0.05)$; PUH: para-umbilical hernia.
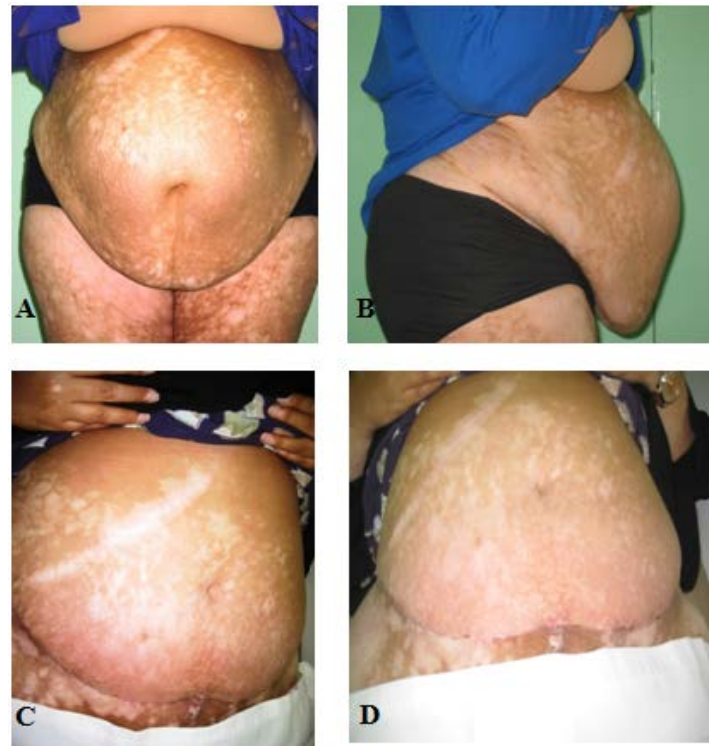

Figure 2. Preoperative views; front (A) and lateral (B), of a 48-year-old obese patient who had given birth to 9 children with severe diastasis of recti and abdominal bulge (grade V), and post-operative views; standing (C) and supine (D), three months after primary hernia repair and meshabdominoplasty
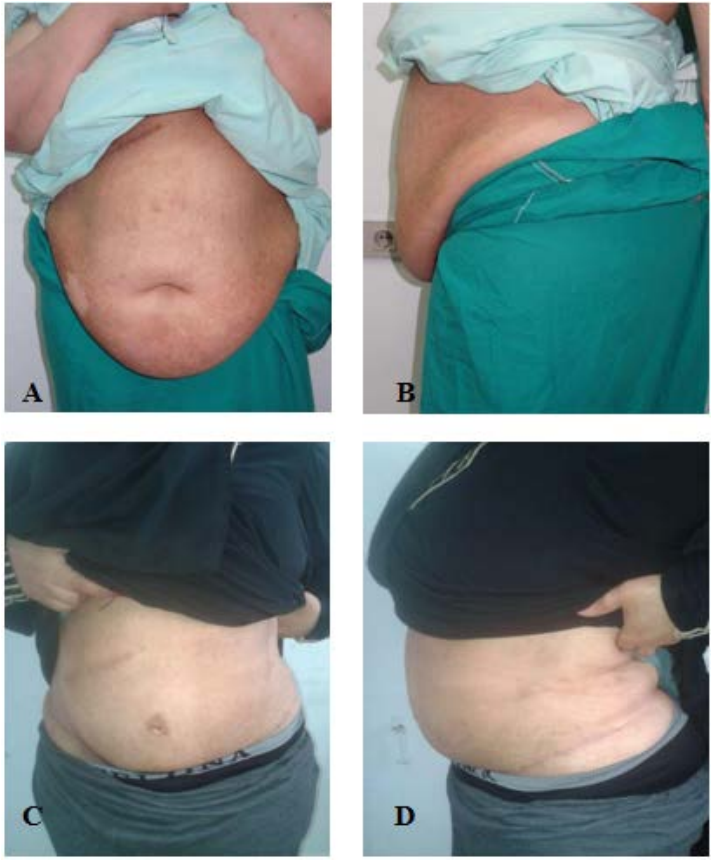

Figure 3. A 42-year-old lady who gave birth to 8 children and had an incisional hernia after previous open cholecystectomy; pre-operative front view (A) and lateral view (B). Post-operative front view (C) and lateral view (D) six months after hernia repair and mesh-abdominoplasty
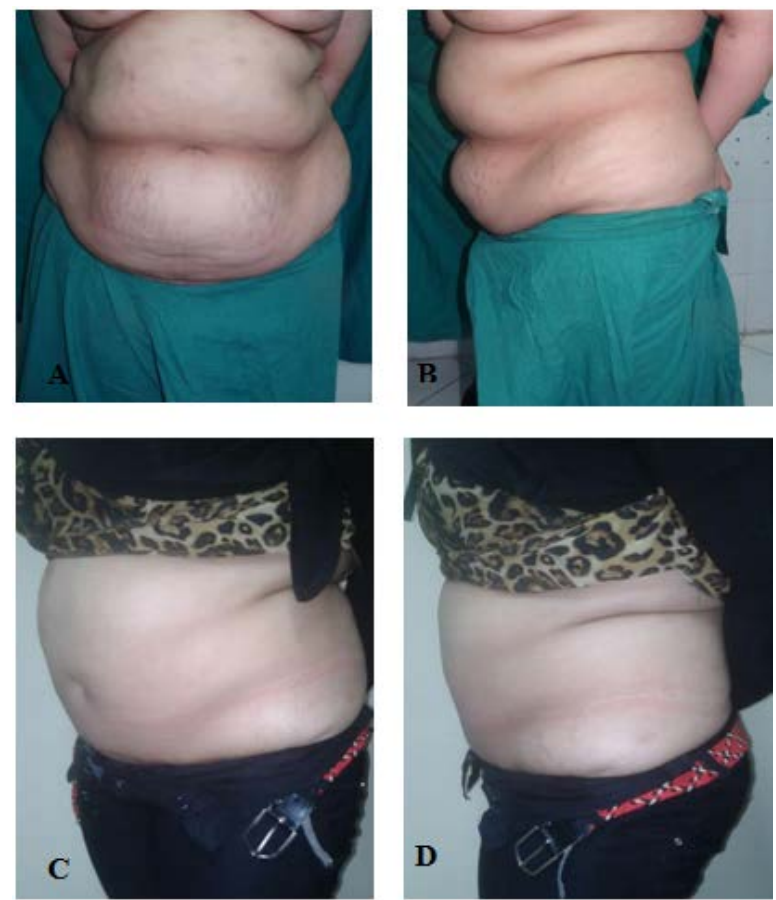

Figure 4. A 38-year-old lady who gave birth to 5 children and had a small epigastric hernia (defect $<3 \mathrm{~cm}$ ); pre-operative front view (A) and lateral view (B). Post-operative oblique view (C) and lateral view (D) 9 months after primary hernia repair and abdominoplasty (without mesh)

Representative cases are shown in Figure 2, Figure 3, Figure 4. All repaired hernias did not recur except for one patient in Group 1 (2.5\%) and another in Group 2 (2.6\%). Abdominal pain was relieved in all patients of both groups. In Group 1 (abdominoplasty), low-back pain was completely relieved in $75.00 \%$ (9/12) of patients and similarly in $70.37 \%(19 / 27)$ of patients in Group 2 (mesh-abdominoplasty) $\quad\left(\mathrm{X}^{2}=0.088, \mathrm{p}=0.767\right)$. It was partially relieved in the rest of patients. No mortality or major complications such as thrombo-embolism, fat embolism, pulmonary comprise, flap necrosis, wound dehiscence or extrusion of the mesh were encountered. Table 3 summarizes post-operative complications encountered in both groups.

Wound complications in the form of hyper-pigmented scar, partial superficial surgical site infection (SSI), and seroma requiring 2-3 percutaneous aspirations occurred in 7 patients (17.5\%) in Group 1 patients versus 10 (26.3\%) in Group $2\left(\mathrm{X}^{2}=0.889, \mathrm{p}=0.346\right)$. In Group 1 patients with abdominoplasty, 15 (37.5\%) required significantly more second operations for refashioning of lower abdominal skin laxity, dog-ears or umbilical scar as compared to 5 patients $(13.2 \%)$ in those with meshabdominoplasty (Group 2) $\left(\mathrm{X}^{2}=6.056, \mathrm{p}=0.0139\right)$. In addition, a second operation (hernioplasty) was required for two patients with recurrent PUHs (in one group each) making a total of 16 patients (40.0\%) versus $6(15.8 \%)$, respectively $\left(\mathrm{X}^{2}=.6 .687, \mathrm{p}=0.001\right)$. Moreover, as seen in Table 4, recurrence of abdominal wall deformity was significantly higher among patients who underwent abdominoplasty alone $\left(\mathrm{X}^{2}=6.461, \mathrm{p}=0.011\right)$. Different grades of abdominal wall recurrence were documented in 23 patients (57.5\%) in Group 1 with no patient suffering from grades IV or $\mathrm{V}$ deformity, versus 11 (28.9\%) in Group 2 patients of whom 6 had grades IV or $\mathrm{V}$ deformity. 
Table 3. Complications of abdominoplasty and meshabdominoplasty in multiparous women with ventral hernia $(\mathrm{N}=78)$

\begin{tabular}{|l|c|c|c|}
\hline Complications & $\begin{array}{c}\text { Abdomino- } \\
\text { plasty } \\
\text { (N=40) }\end{array}$ & $\begin{array}{c}\text { Mesh- } \\
\text { abdomino-plasty } \\
\text { (N=38) }\end{array}$ & $\begin{array}{c}\text { Test of } \\
\text { significance }\end{array}$ \\
\hline $\begin{array}{l}\text { Wound } \\
\text { complications }\end{array}$ & $\mathbf{7 ( 1 7 . 5 )}$ & $\mathbf{1 0}(\mathbf{2 6 . 3 )}$ & \\
$\begin{array}{l}\text { Hyper-pigmented } \\
\text { scar }\end{array}$ & $3(7.5 \%)$ & $6(15.8 \%)$ & $\mathrm{X}^{2}=0.889$ \\
$\begin{array}{l}\text { Superficial SSI } \\
\text { Seroma (requiring } \\
\text { aspiration) }\end{array}$ & $2(5.0 \%)$ & $1(2.6 \%)$ & $\mathrm{P}=0.346$ \\
\hline $\begin{array}{l}\text { Need for 2 } \\
\text { surgery }\end{array}$ & $\mathbf{1 6}(\mathbf{4 0 . 0 \% )}$ & $\mathbf{6}(\mathbf{1 5 . 7 8 \% )}$ & \\
$\begin{array}{l}\text { For recurrence of } \\
\text { abdominal wall } \\
\text { laxity }\end{array}$ & $8(20.0 \%)$ & $2(5.3 \%)$ & $\mathrm{X}^{2}=6.687$ \\
$\begin{array}{l}\text { For "dog ears" } \\
\begin{array}{l}\text { For umbilical scar } \\
\text { revision }\end{array}\end{array}$ & $4(10.0 \%)$ & $2(5.3 \%)$ & $\mathrm{P}=0.001^{*}$ \\
$\begin{array}{l}\text { For recurrent } \\
\text { hernia (PUH) }\end{array}$ & $3(7.5 \%)$ & $1(2.6 \%)$ & \\
\hline
\end{tabular}

* $(\mathrm{p}<0.05$; SSI: surgical site infection, PUH: para-umbilical hernia.

Table 4. Recurrence of abdominal wall deformity [13] after abdominoplasty and mesh-abdominoplasty in multiparous women with ventral hernia $(\mathrm{N}=78)$

\begin{tabular}{|c|c|c|c|}
\hline Grade Description & $\begin{array}{l}\text { Abdomino- } \\
\text { plasty } \\
(\mathrm{N}=40)\end{array}$ & $\begin{array}{c}\text { Mesh- } \\
\text { abdomino- } \\
\text { plasty }(\mathrm{N}=38)\end{array}$ & $\begin{array}{c}\text { Test of } \\
\text { significance }\end{array}$ \\
\hline I: Epigastric bulge. & 7 (17.5\%) & $5(13.1 \%)$ & \multirow{6}{*}{$\begin{array}{l}X^{2}=6.461 \\
P<0.011^{*}\end{array}$} \\
\hline $\begin{array}{l}\text { II: Mild abdominal } \\
\text { wall convexity }\end{array}$ & $6(15.0 \%)$ & $4(10.5 \%)$ & \\
\hline $\begin{array}{l}\text { III: Moderate } \\
\text { abdominal wall } \\
\text { convexity }\end{array}$ & $5(12.5 \%)$ & $2(5.3 \%)$ & \\
\hline $\begin{array}{l}\text { IV: Severe } \\
\text { abdominal wall } \\
\text { convexity }\end{array}$ & $3(7.5 \%)$ & 0 & \\
\hline $\begin{array}{l}\text { V: Total recurrence } \\
\text { of musculo- } \\
\text { aponeurotic laxity }\end{array}$ & $2(5.0 \%)$ & 0 & \\
\hline Total & 23 (57.5\%) & $11(28.9 \%)$ & \\
\hline
\end{tabular}

* Statistically significant $(\mathrm{p}<0.05)$.

\section{Discussion}

In addition to having a diagnosed ventral hernia from whatever cause, multiparous women with severe musculo-aponeurotic laxity, abdominal striae, diastasis recti and/or abdominal wall deformity are usually interested in combining hernia surgery with abdominoplasty. While the need for an operation may be driven by the hernia repair, both are two distinct operations though they are performed in the same area and address problems that often have a common origin. Hernia repair, whether open or laparoscopic, does not do anything for the appearance of the abdominal wall. The goal is just to repair the herniated area. On the other hand, abdominoplasty, which is a cosmetic procedure, is concerned with providing a durable correction of the abdominal wall deformity. Combining the two operations may be reasonable in terms of combining cost and recovery time.

In the present study, patients with a ventral hernia and a defect $<3 \mathrm{~cm}$ (Group 1) were repaired with primary non-absorbable sutures based on the type of repair deemed necessary, and in all cases midline aponeurotic reinforcement was performed. When the defect was $>3$ cm (Group 2) a prosthetic Prolene mesh was applied in addition. According to this protocol, hernia recurrence was similar in both groups being recorded in only one patient in each.

In accordance with our policy and results, several authors reported that primary ventral hernia repair can be performed with a low recurrence rate in patients who have a defect of $<2 \mathrm{~cm}$ for incisional hernia [9], $<3 \mathrm{~cm}$ for PUH [14], <2-3 cm for epigastric hernia [15], or $<4 \mathrm{~cm}$ for ventral hernia in general provided they have rectus abdominis plication performed reinforcing the repair [16]. However, given predisposing factors for recurrence, such as obesity, smoking, steroid usage, or advanced age, the use of a prosthetic material has been recommended even in smaller fascial defects [16]. If defects are $>4 \mathrm{~cm}$, they should be repaired with a prosthesis, unless an associated infection or other source of contamination is present $[17,18]$. In addition, similar to our strategy, Cheesborough et al [7], who studied the outcome of simultaneous meshabdominoplasty for large ventral hernia and severe rectus diastasis repairs, reported no hernia or bulge recurrences with a mean follow-up of 16 months. It is noteworthy that the high recurrence rate, reaching $10 \%-50 \%$ [15], reported in the literature for ventral hernias with defects of $<4 \mathrm{~cm}$ was noted when reinforcement with midline aponeurotic plication was not performed. Other authors [17,18], reported even a higher hernia recurrence of $67 \%$ with primary closure alone in large defects of $>10 \mathrm{~cm}$, and with such large defects, they recommended reinforcement with a prosthetic mesh.

The concern when repairing ventral hernias during abdominoplasty is related to the blood supply of the umbilicus, which has a dual blood supply - from its base and from the skin. When dissecting the abdominal flap in standard abdominoplasty, the umbilical stalk is detached from the skin flap leaving vascularization of the umbilicus to be provided only by vessels entering its base from the musculo-aponeurotic layer. At the same time, ventral hernia repair via direct closure or mesh may require incision at the umbilical stalk base, which may jeopardize the umbilical perforating vessels and result in partial or full umbilical necrosis with possible infection of the prosthesis [17]. In order to minimize the risk of potential devascularization of the umbilical stalk several approaches have been proposed for simultaneous abdominoplasty and ventral hernia repair via the open approach with or without mesh. McKnight et al [17], reported on the "right lateral" approach in which they preserve the rectus muscle sheet completely on one side. A similar approach was reported by Bruner et al [19], but via an inferior midline incision in 17 patients without umbilical necrosis. Neinstein et al [18], used a Ventralex patch via open approach in 11 patients without vascular compromise. Kulhanek and Mestak [20] performed the hernia repair via open approach but the umbilicus stayed attached to the skin. Other techniques described to preserve umbilical blood supply, including ours in some cases, entail fascial incisions away from the umbilical stalk and sacrificing the umbilicus. Al-Shaham [21] described sacrificing the natural umbilicus followed by neo-umbilicoplasty being needed when short umbilical stumps are encountered or an associated umbilical hernia is present along with wide myo-fascial plication distances $(12-19 \mathrm{~cm})$. 
Recently, in 2017, Hristo et al [22] have associated the abdominoplasty with umbilical hernia repair via laparoscopy; thus, obviating any incision near the umbilical stalk base. However, a patient with a history of abdominal surgery and possible complications from adhesions may not be a candidate for laparoscopic surgery. Moreover, it is an invasive intra-peritoneal approach, which is technically demanding, more expensive and time-consuming than the open approach. Furthermore, a longer follow-up is definitely required to reach solid conclusions about its results.

The addition of abdominoplasty at the time of ventral hernia repair may increase the rate of wound complications particularly when mesh is involved. Despite severe musculo-aponeurotic laxity in our multiparous patients, extensive abdominal contouring technique, addition of liposuction and the application of a Prolene mesh to reinforce the midline plication in Group 2 patients, no major complications were encountered. Moreover, the occurrence of wound complications reported herein was comparable in both groups, which correlates with the results reported in the literature [23,24,25] including multiparous women [13], the use of stapled Prolene mesh [26], or the application of a submuscular resorbable mesh [27]. The use of Prolene mesh in the present study did not add to the occurrence of post-operative seroma, which was recorded in $6.41 \%$ of patients, which coincides with the $3 \%-7 \%$ reported post-abdominoplasty by several authors $[7,28]$. All seromas in our patients resolved after 2-3 percutaneous aspirations under aseptic conditions in the outpatient clinic. Horndeski and Gonzalez [29] who performed abdominoplasty with mesh reinforcement ventral hernioplasty for 14 patients reported two seromas only. Downey et al [30] reported that seroma developed in 20 of their 50 patients of whom six required aspiration(s). However, their population was different from ours, since all their patients had incisional hernia and post-bariatric abdominoplasty. Likewise, Harth et al [31] documented a five-fold increased incidence of wound-related morbidity when abdominoplasty was combined with a mesh-based hernia repair; however, all their patients, unlike ours, were morbidly obese. Allyson et al [9] reported similar SSI rate among patients with primary suture repairs for ventral hernia and those with synthetic mesh repairs $(6.6 \%$ vs 7.3\%, respectively). Although a sublay location for mesh placement during ventral hernia repair is preferred, development of the retro-rectus space, risks damage to the umbilical perforators, particularly when combined with an abdominoplasty procedure [32].

In this study, the use Prolene mesh (Group 2) had a dual benefit; reducing hernia recurrence and providing a sustainable correction of abdominal wall deformity. In multiparous patients with severe musculo-aponeurotic laxity who underwent standard abdominoplasty, central plication alone has been reported to be insufficient in preserving a durable waist improvement and body contour [10,27,33-38]. Patients who underwent meshabdominoplasty (Group 2) in the current study had significantly lower recurrence rate of abdominal deformity and less need for secondary procedures as compared to those who underwent abdominoplasty alone (Group 1). In accordance with our findings, Hickey et al [27], noted high recurrence of deformity after plication alone during abdominoplasty reaching up to $40 \%$. In addition,
Al-Qattan [13] reported that all 20 multiparous patients with severe musculo-aponeurotic laxity repaired with midline plication alone, had partial recurrence of their laxity at an average follow-up of one year. In this particular group of patients, the use of Marlex mesh for plication of abdominoplasty in a study by Marques et al [38] provided a marked degree of musculo-aponeurotic reinforcement without any infection, dehiscence, or extrusion of the mesh in any of the 18 patients reviewed. Similarly, a suture midline plication followed by fixation of a Prolene mesh with 2/0 Prolene sutures provided a safe and reliable technique with no associated reports of major complications [26], which is consistent with the results presented in this study utilizing a similar technique.

Regarding secondary procedures, Hensel et al [1], reviewed retrospectively 199 abdominoplasty patients during a 15 -year period and reported a $43 \%$ revision rate; however, it was mainly related to fine-tuning the aesthetic appearance. Al-Qattan [13] reported that one-third of their patients required a second procedure for umbilical reconstruction and revision of partial recurrence [13], which is comparable to the $37.5 \%$ (15/40) of our patients who required refashioning surgery after abdominoplasty alone. Moreover, only 13.16\% (5/38) of our patients who underwent mesh-abdominoplasty required refashioning surgery. In agreement with our findings, Kaddoura and Nasser [26], who used a stapled Prolene mesh for reinforcement of midline abdominal wall plication in 21 multiparous patients with severe musculo-aponeurotic laxity reported that 4 patients (19\%) underwent umbilical and dog ear revisions.

From the results of this study, it may be concluded that (1) during an abdominoplasty, midline fascial plication and ventral hernia repair can be performed in defects $<3$ $\mathrm{cm}$ without mesh reinforcement with no increase in hernia recurrence rates, (2) in patients with defects $>3 \mathrm{~cm}$, mesh reinforcement is indicated after suture hernia repair and midline plication, and (3) the use of Prolene mesh in abdominoplasty for multiparous women with severe musculo-aponeurotic laxity and ventral hernia, yields lower recurrence of abdominal deformity and less refashioning procedures with minimal complications than abdominoplasty alone.

\section{References}

[1] Hensel JM, Lehman JA, Tantri MP, et al. An outcome analysis and satisfaction survey of 199 consecutive abdominoplasties. Ann Plast Surg 2001; 46 (4): 357-63.

[2] Haritopoulos KN, Labruzzo C, Paplios VE, et al. Abdominoplasty in a patient with severe obesity. Int Surg 2002; 87 (1): 15-18.

[3] Sesung-Jun O, Thaller SR. Refinements in abdominoplasty. Clin Plast Surg 2002; 29 (1): 95-109.

[4] Pratt JH, Irons GB. Panniculectomy and abdominoplasty. Am J Obstet Gynecol 1978; 132 (2): 165-168.

[5] Grazer FM, Goldwyn RM. Abdominoplasty assessed by survey with emphasis on complications. Plast Reconstr Surg 1977; 59 (4): 513-517.

[6] Psillakis JM. Abdominoplasty: some ideas to improve results. Aesth Plast Surg 1978; 2: 205-215.

[7] Cheesborough JE, Gregory A. Dumanian GA. Simultaneous Prosthetic Mesh Abdominal Wall Reconstruction with Abdominoplasty for Ventral Hernia and Severe Rectus Diastasis Repairs. Plast Reconstr Surg 2015; 135(1): 268-276.

[8] Baxter RA. Controlled results with abdominoplasty. Aesthetic plastic surgery 2001; 25 (5): 357-364. 
[9] Moulton AW, Branman R. Incidental Anterior Abdomina Hernias during Abdominoplasty: An Algorithmic Approach to Repair. AJCS 2016; 33(3): 121-127.

[10] Bozola AR, Psillakis JM. Abdominoplasty: a new concept and classification of treatment. Plast Reconstr Surg 1988; 82 (6): 983-993.

[11] Dubou R, Ousterhout DK. Placement of the umbilicus in an abdominoplasty. Plast Reconstr Surg 1978; 61: 291-293.

[12] Abhyankar SV, Rajguru AG, Patil PA. Anatomical localization of the umbilicus: an Indian study. Plast Reconstr Surg 2006; 117: 1153-1157.

[13] Al-Qattan MM. Abdominoplasty in multiparous women with severe musculoaponeurotic laxity. Br J Plast Surg 1997; 50: 450-454.

[14] Arshava E. Open repair of ventral hernias. In: Donnellan K. Essential Operative Techniques and Anatomy. Philadelphia, PA: Lippincott Williams \& Wilkins, A Wolters Kluwer Business; 2014: 253.

[15] Squires R. Ventral abdominal hernias. In: Townsend CM. ed. Sabiston Textbook of Surgery: The Biological Basis of Modern Surgical Practice. Philadelphia, PA: Saunders Publishing; 2012: 1155-1179.

[16] Fitzsgibbons R Jr. Open hernia repair. In: Cameron J, ed. American College of Surgery, Surgery: Principles \& Practice. Chicago, IL: The University of Chicago/B.C. Decker; 2007: 828-849.

[17] McKnight C, Fowler JL, Cobb WS, Smith DE, Carbonell AM. Concomitant sublay mesh repair of umbilical hernia and abdominoplasty. Can J Plast Surg 2012; 20(4): 258-260.

[18] Neinstein RM, Matarasso A, Abramson DL. Concomitant abdominoplasty and umbilical hernia repair using the Ventralex hernia patch. Plast Reconstr Surg. 2015; 135(4): 1021-1025.

[19] Bruner TW, Salazer-Reyes H, Friedman JD. Umbilical hernia repair in conjunction with abdominoplasty: a surgical technique to maintain umbilical blood supply. Aesthetic Surg J 2009; 29: 333-334.

[20] Kulhanek J, Mestak O. Treatment of umbilical hernia and recti muscles diastasis without a periumbilical incision. Hernia 2013; 17: 527-530.

[21] Al-shaham A. Neoumbilicoplasty is a useful adjuvant procedure in abdominoplasty. Can J Plast Surg. 2009; 17(4): e20-e23.

[22] Shipkov HD, Mojallal A, Braye F. Simultaneous Abdominoplasty and Umbilical Hernia Repair via Laparoscopy: a Preliminary Report. Folia Medica 2017; 59 (2): 222-227.
[23] Hafezi F, Nouhi A. Safe abdominoplasty with extensive liposuctioning. Ann Plast Surg. Aug 2006; 57(2): 149-153.

[24] Zdravka B, Nicolas L, Christophe L, Patrick L. Use of submuscular resorbable mesh for correction of severe musculoaponeurotic laxity: an 11-year retrospective study.

[25] Regnault P. Abdominal dermolipectomies. Clin Plast Surg 1975; 2(3): 411-429.

[26] Kaddoura IL, Nasser A. Abdominoplasty: The use of stapled prolene mesh in severe musculo-aponeurotic laxity. Aesthetic Surgery Journal 1998; 18 (5): 335-341.

[27] Hickey F, Finch JG, Khanna A. A systematic review on the outcomes of correction of diastasis of the recti. Hernia 2011; 15: 607-614.

[28] van Uchelen JH, Kon M, Werker PM. The long-term durability of plication of the anterior rectus sheath assessed by ultrasonography. Plast Reconstr Surg 2001; 107: 1578-1584.

[29] Horndeski G, Gonzalez E. Abdominoplasty with mesh reinforcement ventral herniorrhaphy. Plast Reconstr Surg 2001; 128: 101e-102e.

[30] Downey SE, Morales C, Kelso RL, et al. Review of technique for combined closed incisional hernia repair and panniculectomy status post open bariatric surgery. Surg Obes Relat Dis 2005; 1: 458-461.

[31] Harth KC, Blatnik JA, Roesn MJ. Optimum repair for massive ventral hernias in the morbidly obese patient - is panniculectomy helpful? Am J Surg 2011; 201: 396-400.

[32] Stoppa R. The treatment of complicated groin and incisional hernias. World J Surg 1989; 13: 545-554.

[33] Appiani E. Muscular plastic for aesthetic conformation of abdominal girdle. Ann Plast Surg 1984; 13 (2): 97-106.

[34] Baroudi R. Body contour surgery. Clin Plast Surg 1989; 16 (2): 263-277.

[35] De Pina DP. Aesthetic abdominal deformities: a personal approach to the posterior rectus sheath and rectus muscles. Plast Reconstr Surg 1985; 75 (5): 660-667.

[36] Abramo AC, Viola JC, Marques A. The H approach to abdominal muscle aponeurosis for the improvement of body contour. Plast Reconstr Surg 1990; 86 (5): 1008-1013.

[37] Baroudi R, Maraes M. Philosophy, technical principles, selection and indication in body contouring surgery. Aesthetic Plast Surg 1991; 15 (1): 1-18.

[38] Marques A, Brenda E, Ishizuka MA, Abramo AC, Andrews JM. Abdominoplasty: modified plication. Br J Plast Surg 1990; 43 (4): 473-475. 